\title{
BDNF Gene Polymorphisms and Motor Cortical Plasticity in Healthy Humans: When Should We Consider It?
}

\author{
Andrea Antal, ${ }^{*}$ Leila Chaieb, ${ }^{3}$ Vera Moliadze, ${ }^{1}$ Driss Bourakkadi Zarrouki, ${ }^{1}$ Moneef Shoukier ${ }^{2}$ \\ and Walter Paulus ${ }^{1}$
}

' Department of Clinical Neurophysiology, Georg-August University of Göttingen, Germany

${ }^{2}$ Institute of Human Genetics, Georg-August University of Göttingen, Germany

${ }^{3}$ Clinic for Epileptology, Germany

${ }^{*}$ CoRresponding AUtHoR:

Andrea Antal

Department of Clinical

Neurophysiology

Georg-August University

Robert-Koch-Str. 40

37075 Göttingen, Germany

Tel: +49-551-398461

Fax: +49-551-398126

E-mail: Aantal@gwdg.de

Received: 06 Jun 2014

Accepted: 30 Sep 2014

Published: 03 Oct 2014

(c)

This article is distributed under the terms of the Creative Commons Attribution License, which permits unrestricted use and redistribution provided that the original author and source are credited.

Competing interests: The authors declare that no competing interests exist.

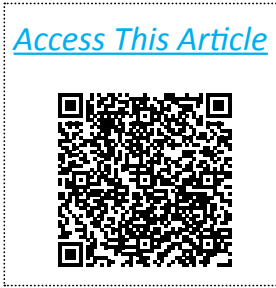

\section{Abstract}

Background: The brain-derived neurotrophic factor (BDNF) gene is involved in mechanisms of synaptic plasticity in the brain and has been demonstrated to also play a role in influencing brain plasticity induced by transcranial magnetic and electrical stimulation.

Objective and methods: This is an update of a previous study from our laboratory. We retrospectively analysed the data of 115 healthy subjects participating in 130 experimental sessions, measuring the amplitude of motor evoked potentials (MEPs) before and after transcranial stimulation of the primary motor cortex (M1). We explored whether BDNF polymorphism shapes the effects of excitatory theta burst stimulation (iTBS, $n=23)$, anodal $(n=32)$ and cathodal $(n=19)$ transcranial direct current (tDCS), random noise (tRNS, $n=33$ ) and alternating current (tACS, $n=13$ ) stimulation.

Results: Although a trend toward altered plasticity was observed in Val$66 \mathrm{Met}$ allele carriers to stimulation with regard to all protocols compared with the response of Val66Val individuals, no significant GENOTYPE $x$ TIME interaction was found.

Conclusions: The BDNF polymorphism is suggested to have an impact on transcranial stimulation-induced plasticity in humans, which differs according to the mechanism of plasticity induction. However, according to our data, we suggest that genotyping in general, in transcranial stimulation studies including small number of subjects and at least when the M1 is stimulated, is not necessary. Nevertheless, the impact of BDNF on plasticity inducing protocols might be taken into account for e.g. in cognitive studies, when the prefrontal cortex is stimulated.

Keywords: Transcranial random noise stimulation (tRNS), Transcranial

Cite as: Antal A, Chaieb L, Moliadze V, et al. BDNF Gene Polymorphisms and Motor Cortical Plasticity in Healthy Humans: When Should We Consider It?. J Neurosci Rehabil. 2014;1 (2):1-13. 
alternating current stimulation (tACS), Transcranial direct current stimulation (tDCS), Transcranial magnetic stimulation (TMS), Theta burst stimulation (TBS), Neuroplasticity, Brain-derived neurotrophic factor (BDNF)

INTRODUCTION

Val66Met (rs6265) is a single nucleotide polymorphism (SNP) in the brain-derived neurotrophic factor (BDNF) gene that codes for the protein BDNF. ${ }^{[1]}$ Many studies have confirmed the role of BDNF in regulating synaptic consolidation in adults and neural plasticity. ${ }^{[2-6]}$ When the substitution of Met for Val occurs at position 66 in the pro-region of the BDNF gene, altered activity-dependent release and recruitment of BDNF in neurons can be observed. ${ }^{[7]}$ This is believed to manifest in an altered ability to induce neuroplasticity in the human brain. Many studies using non-invasive neuroplasticity-inducing protocols have investigated the impact of the BDNF polymorphism on cortical modulation and plasticity, usually by investigating the excitability alterations in the primary motor cortex (M1) induced by different transcranial stimulation methods. ${ }^{[8-13]}$ However, the results are partly contradictory and mainly dependent on the paradigm used in a given study. Originally ${ }^{[8]}$ for intermittent theta burst stimulation (iTBS), continuous theta burst stimulation (cTBS), paired associative stimulation (PAS) and combined transcranial direct current stimulation/ repetitive transcranial magnetic stimulation (tDCS/rTMS) metaplasticity (e.g. $1 \mathrm{~Hz}$ rTMS) protocols, Met carriers showed a reduced response to iTBS cTBS and to PAS protocols compared to Val66Val individuals. This was essentially confirmed later in a retrospective study ${ }^{[9]}$ reporting that Val66Val individuals showed larger MEP responses than Val66Met subjects after iTBS. In contrast, other studies observed that the aftereffects of $i \mathrm{TBS}^{[10]}$ or quadripulse stimulation (QPS) ${ }^{[12]}$ could not be modulated by the BDNF Val66Met polymorphism. Similarly, no difference in homeostatic metaplasticity between Val66Val participants and Met carriers in a three-session, repeated measures (cTBS followed by cTBS, cTBS followed by iTBS, and iTBS followed by iTBS) paradigm ${ }^{[11]}$ was seen. For tDCS, the aftereffects of 7-10min anodal and cathodal stimulation on MEP amplitudes was shown to be greater for Met carriers, but this finding was not statistically significant. ${ }^{[9]}$ This was confirmed by another study using a 20 min stimulation duration ${ }^{[14]}$ and also in the case of transcranial random noise stimulation (tRNS). ${ }^{[9]}$

The results summarized above suggest that Met carriers might be less likely to undergo neuroplastic changes using some of the stimulation protocols. It is possible that the Met carriers are the 'non-responders', who are commonly attributed to their unability to relax during measurements in each laboratory using transcranial stimulation methods. The question rises, whether we should always consider this genetical factor, when we are using transcranial stimulation in a laboratory environment. Here we have updated our previous observations ${ }^{[9]}$ in a larger subject population including 115 healthy subjects and 130 measurements and again, using a retrospective approach with participants grouped according to genotype. Besides the increase in the number of subjects we included data using transcranial alternating current stimulation (tACS). The results still suggest some trends toward specific plasticity induction protocols being dependent on BDNF polymorphisms, however, without statistical significance.

\section{Material and Methods}

\section{Subjects}

Altogether the data of 115 healthy volunteers (age range: 19-40 years) participating in 130 experimental sessions was analysed (Table. 01). Subjects were included in this retrospective analysis study after giving written informed consent. None of them suffered from any neurological or psychological disorders, none had metallic implants or implanted electric devices or took any medication prior to the experimental sessions or on a regular

Cite as: Antal A, Chaieb L, Moliadze V, et al. BDNF Gene Polymorphisms and Motor Cortical Plasticity in Healthy Humans: When Should We Consider It?. J Neurosci Rehabil. 2014;1 (2):1-13. 
basis. We conform to the Declaration of Helsinki and the retrospective analysis was approved by the Ethics Committee of the University of Göttingen.

\section{Experimental procedure}

Healthy subjects who had participated in our previous experiments using transcranial stimulation methods were asked to participate in the BDNF genotyping study. ${ }^{[9,15-21]}$ Unfortunately not all the subjects could be contacted, and a few refused to participate in the genotyping study. In addition, not all of the subjects participated in all of the stimulation conditions. Therefore the number of subjects in the different experimental sessions varies (Table. 01). After successful genotyping, the previously recorded MEP data from Val66Val homozygotes and Val66Met heterozygotes were identified and analysed.

\begin{tabular}{|c|c|c|}
\hline Stimulation & Genotype & Subjects \\
\hline \multirow[t]{2}{*}{ a-tDCS } & 19 Val66Val & $11 \mathrm{~F} / 8 \mathrm{M}$ \\
\hline & 13Val66Met & $8 \mathrm{~F} / 5 \mathrm{M}$ \\
\hline \multirow[t]{2}{*}{ c-tDCs } & $11 \mathrm{Val66Val}$ & $5 F / 6 M$ \\
\hline & 8Val66Met & $4 \mathrm{~F} / 4 \mathrm{M}$ \\
\hline \multirow[t]{2}{*}{ tACS } & $7 \mathrm{Val} 66 \mathrm{Val}$ & $5 F / 2 M$ \\
\hline & 6Val66Met & $1 F / 5 \mathrm{M}$ \\
\hline \multirow[t]{2}{*}{ tRNS } & $30 \mathrm{Val} 66 \mathrm{Val}$ & $19 \mathrm{~F} / 11 \mathrm{M}$ \\
\hline & 13Val66Met & $10 F / 3 M$ \\
\hline \multirow[t]{2}{*}{ iTBS } & $15 \mathrm{Val} 66 \mathrm{Val}$ & $10 \mathrm{~F} / 5 \mathrm{M}$ \\
\hline & 8 Val66Met & $6 \mathrm{~F} / 2 \mathrm{M}$ \\
\hline
\end{tabular}

Table. 01 Table illustrates the number of participants and their genotypes in each condition.

\section{BDNF genotyping}

Whole blood was vacuum extracted into EDTA tubes and DNA was sampled using standard laboratory procedures. Primer sequences and PCR conditions are available upon request from M. Shoukier). PCR analysis was checked for positive sequence markers on agarose $/ 2 \times$ TBE gels. Restriction Fragment Length Polymorphism (RFLP) analysis was performed by digesting the PCR product with the restriction enzyme Hsp92II. RFLP-conditions are available upon request. Restriction products were then electrophoresed on a $2 \%$ agarose gel and visualized using a transilluminator and ethidium bromide staining. After digestion the Val allele (G) gave two fragments: 57 and $217 \mathrm{bp}$, whereas the Met allele (A) gave three: 57, 77 and $140 \mathrm{bp}$. The 115 participants were genotyped as follows: 69 participants were found to be homozygous for the Val allele (Val66Val), 40 were Val66Met heterozygotes and 6 were homozygous for the Met allele. The Met homozygotes were not included in this analysis. The blood sampling was done on non-experimental days, and the period between the last experimental session and the taking of the blood sample was between 1 day and 2 months.

\section{MEP measurement}

The MEP registration protocols are described in detail in previous publications. ${ }^{[9,15-21]}$ Briefly, subjects were seated in a comfortable reclining chair with a mounted headrest throughout the experiments. Within each type of

Cite as: Antal A, Chaieb L, Moliadze V, et al. BDNF Gene Polymorphisms and Motor Cortical Plasticity in Healthy Humans: When Should We Consider It?. J Neurosci Rehabil. 2014;1 (2):1-13. 
experimental session the measurements were always performed by the same experienced investigator. To detect current-driven changes in cortical excitability, MEPs of the right ADM or FDI were recorded following stimulation of its motor-cortical representation using single-pulse TMS. These were elicited using a Magstim 200 or using MagPro magnetic stimulator, with a figure-of-eight standard magnetic coil (diameter of one winding, $70 \mathrm{~mm}$ ). Surface electromyography (EMG) was recorded from the right FDI and ADM muscle through a pair of $\mathrm{Ag}-\mathrm{AgCl}$ electrodes in a belly-tendon montage. Raw signals were amplified, band-pass filtered $(2 \mathrm{~Hz}-3 \mathrm{kHz}$; sampling rate $5 \mathrm{kHz}$ ), digitized with a micro $1401 \mathrm{AD}$ converter (Cambridge Electronic Design, Cambridge, UK) controlled by Signal Software (version 2.13). Complete relaxation was controlled through visual feedback of EMG activity. The coil was held tangentially to the skull, with the handle pointing backwards and laterally at $45^{\circ}$ from the midline, resulting in a posterior-anterior current flow direction in the brain. The optimum position was defined as the site where TMS resulted consistently in the largest and most stable MEP in the resting muscle. The intensity required to evoke a MEP of $\sim 1 \mathrm{mV}$ peak-to-peak amplitude (SI1mV) and a baseline of TMS-evoked MEPs (40 stimuli) were recorded at $0.25 \mathrm{~Hz}$ prior to stimulation. Following plasticity-inducing stimulation, 40 single test-pulse MEPs were recorded at $0.25 \mathrm{~Hz}$, i.e. approximately at 0 min and then every 5 or 10 minutes up to 60 min post-stimulation min, depending on the experimental protocol. As different investigators measured MEPs at different time points post-stimulation, we have chosen four common timepoints for the purpose of this analysis: baseline (MEPs recorded prior to stimulation) and measurements taken between 0-5, 10-15, 25-30, 55-60 min post-stimulation.

\section{Electrical and magnetic stimulation: tRNS, tDCS, tACS and iTBS}

Electrical stimulation was delivered using a battery-driven electrical stimulator (DC-Stimulator-Plus, NeuroConn $\mathrm{GmbH}$, Ilmenau, Germany) through conductive-rubber electrodes, placed in two saline-soaked sponges. The stimulation electrode was placed over the left M1, which was determined by single-pulse TMS. The reference electrode was placed over the contralateral orbit. The stimulation intensity was $1 \mathrm{~mA}$. The detailed description of the stimulation conditions can be found in previous publications. ${ }^{[9,15-21]}$

In summary:

tRNS - ( $n=43 ; 13$ heterozygotes): In the stimulation mode "noise" there is a random output of current generated for every sample. ${ }^{[15]}$ The random numbers are normally distributed; the probability density function follows a bell-shaped curve. In the frequency spectrum all coefficients have a similar size. The noise signal contains all frequencies up to half of the sampling rate, i.e. a maximum of $640 \mathrm{~Hz}$. The size of the stimulation electrode was $4 \times 4 \mathrm{~cm}$ and the reference electrode was $6 \times 14 \mathrm{~cm}$. TRNS was applied for 10 minutes.

tDCS - ( $n=32 ; 32$ anodal stimulation; 13 heterozygotes and 19 cathodal stimulation, 8 heterozygotes): Here the electrode size was $5 \times 7 \mathrm{~cm}$. Stimulation was performed for 7 (2 subjects) 10 (25 subjects) or 13 ( 5 subjects) and 9 (2 subjects) or 10 (17 subjects) minutes (for anodal and cathodal stimulation polarities respectively, according to the different protocols from each study).

tACS - $(n=13 ; 6$ heterozygotes): tACS was applied for 10 min with a current intensity of $1 \mathrm{~mA}$ and with a frequency of $140 \mathrm{~Hz}$. The waveform of the stimulation was sinusoidal without a DC offset. The current was ramped up and down over the first and last $5 \mathrm{~s}$ of stimulation. Here the electrode size over the M1 was $4 \times 4 \mathrm{~cm}$ whereas the reference electrode size at the forehead was $14 \times 6 \mathrm{~cm}$.

iTBS - $(n=22 ; 8$ heterozygotes): iTBS was applied using a Magstim Super Rapid (Magstim Company, Whiteland, Wales, UK) with a standard, figure-of-eight-coil and MagPro stimulator (Medtronic, Denmark) with an outer half-coil radius of $75 \mathrm{~mm}$, with a posterior-anterior-posterior current flow in the coil. Stimulus intensity

Cite as: Antal A, Chaieb L, Moliadze V, et al. BDNF Gene Polymorphisms and Motor Cortical Plasticity in Healthy Humans: When Should We Consider It?. J Neurosci Rehabil. 2014;1 (2):1-13. 
was $80 \%$ of active motor threshold (AMT). AMT was defined as the lowest stimulus intensity at which three of six consecutive stimuli elicited reliable MEPs $(\sim 200 \mu \mathrm{V}$ in amplitude) in the tonically contracting the right first dorsal interosseous (FDI) or the abductor digiti minimi (ADM) muscle. The pattern of iTBS consisted of bursts of stimuli containing 3 pulses at $50 \mathrm{~Hz}$ repeated at $200 \mathrm{~ms}$ intervals (i.e., at $5 \mathrm{~Hz}$ ). A 2s train of TBS was repeated every 10 s for a total of $190 \mathrm{~s}$ (600 pulses).

\section{Data analyses}

In order to estimate the numbers of subjects needed in each group power analysis were done based on the results of previously published data ${ }^{[8,9]}$ (with regard to tRNS and tACS there are no data available). In order to detect the difference in the mean MEP size between Val/Val and Val/Met subjects with $95 \%$ confidence and $80 \%$ power, in the iTBS group min. 8 , in the tDCS group 11 Val66Met subjects should be included.

Student's t-test was used to compare baseline with post-stimulation MEP amplitudes within one condition and considering only one genotype, in order to see if the given stimulation condition had an effect. After it repeated measures ANOVAs with the between subject factor GENOTYPE, the within subject factor TIME (before, 0-5, 10-15, 25-30, 55-60 min post-stimulation) and the dependent variable MEP amplitude were used to compare the effects of neuroplasticity-induction and different stimulation methods among individuals. Effects were considered significant if $p<0.05$. The results were not Bonferroni corrected for multiple testing. All data are given as means + SEM.

RESULTS

iTBS: The raw MEP data from 23 individuals are plotted in Figure. 01. Compared to the baseline MEPS, there was a significant increase in MEP amplitude after iTBS in the Val66Val group during the first 30 minutes $(2.99<\mathrm{t}>3.2, \mathrm{p}<0.01)$, but not in the Val66Met group.

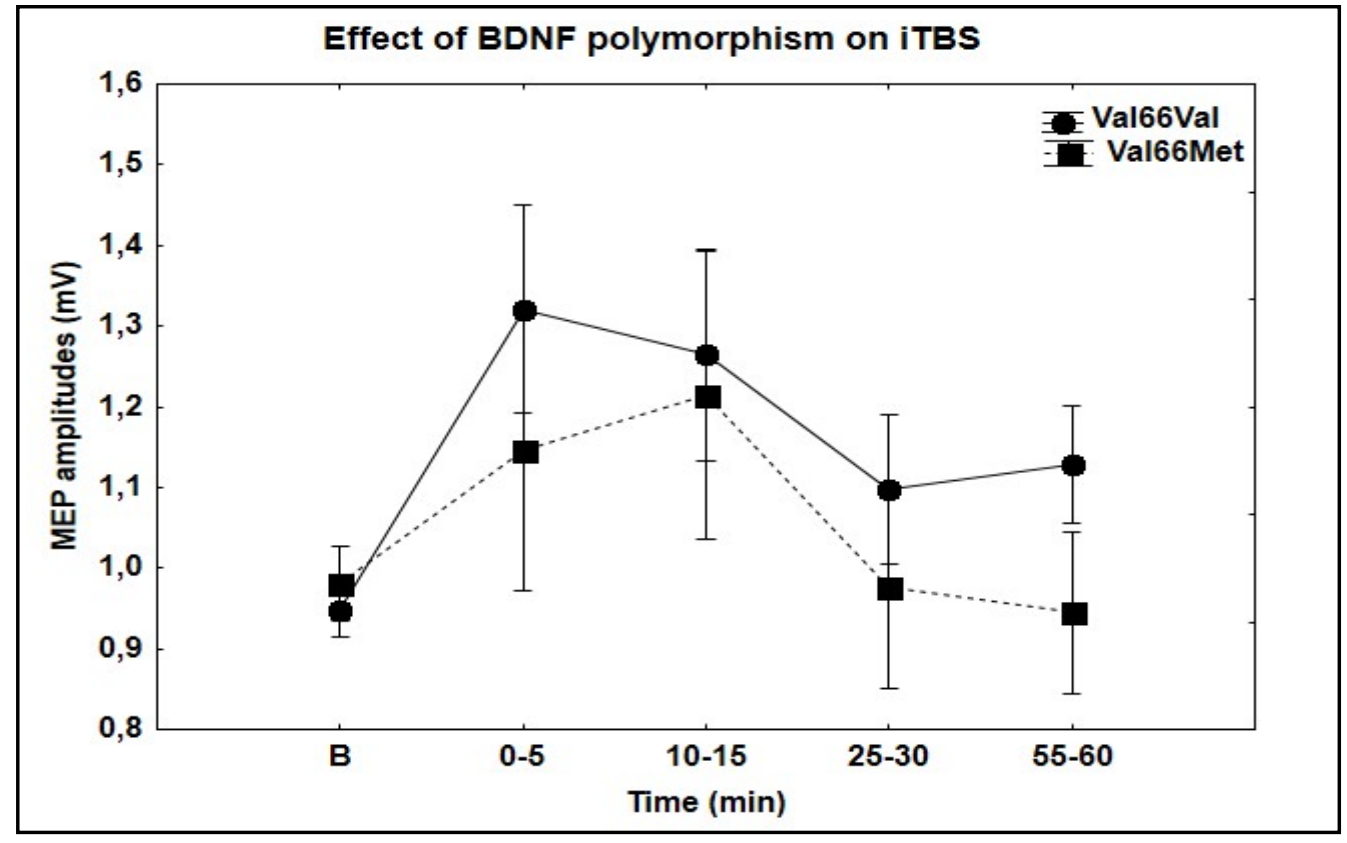

Figure. 01 Effect of BDNF polymorphism on iTBS

Cite as: Antal A, Chaieb L, Moliadze V, et al. BDNF Gene Polymorphisms and Motor Cortical Plasticity in Healthy Humans: When Should We Consider It?. J Neurosci Rehabil. 2014;1 (2):1-13. 
Figure. 01 Effect of BDNF Val66Met polymorphism on cortical excitability in response to iTBS. There was a significant increase in MEPS after iTBS in the Val66Val group, but not in the Val66Met group. Data are mean (+ S.E.M.) peak-to-peak amplitudes of MEP.

The ANOVA revealed no significant main effect of GENOTYPE $\left(F_{1,12}=0.57 ; p=0.45\right)$ and GENOTYPE x TIME interaction $\left(F_{4,84}=0.61 ; p=0.65\right)$. The effect of TIME was significant $\left(F_{4,84}=4.71 ; p=0.002\right)$.

tDCS: With regard to anodal stimulation there was a significant increase in MEP size after anodal tDCS in both groups compared to the baseline MEPs but the excitability enhancement was more pronounced in the Val66Met group (Val66Val group: during the first $30 \mathrm{~min} 2.57<\mathrm{t}>3.91, \mathrm{p}<0.02$; Val66Met group: up to $60 \mathrm{~min}$ $3.12<\mathrm{t}>5.61, \mathrm{p}<0.01$ ) (Figure. 02).

The ANOVA revealed no significant main effect of GENOTYPE $\left(F_{1,30}=2.99 ; p=0.09\right)$, and GENOTYPE $\times$ TIME interaction $\left(F_{4,120}=2.12 ; p=0.08\right)$. The effect of TIME $\left(F_{4,120}=6.42 ; p=0.0001\right)$, however, was significant.

With regard to cathodal stimulation when the post-stimulation MEP amplitudes were compared to baseline MEP values, the induced cortical inhibition was more pronounced in the Val66Met group than in the Val66Val group (Val66Val group: $=$ and $10 \mathrm{~min} 2.38<\mathrm{t}>7.72, \mathrm{p}<0.03$; Val66Met group: during the first $30 \mathrm{~min} 3.38<\mathrm{t}>6.94$, $\mathrm{p}<0.01$ ) (Figure. 02).

The ANOVA revealed no significant main effect of GENOTYPE $\left(F_{1,17}=0.93 ; p=0.34\right)$, however the effect of TIME was significant $\left(F_{4,68}=9.57 ; p=0.0001\right)$. The GENOTYPE $x$ TIME interaction was not significant $\left(F_{4,68}=1.45\right.$; $p=0.22$ ).

tRNS: The t-test showed that there was a significant increase in MEP amplitude after tRNS in both groups at all timepoints compared to the baseline (Val66Val group: 3.86<t>5.3, $\mathrm{p}<0.004$; Val66Met group: $2.50<\mathrm{t}>4.1$, $\mathrm{p}<0.03$ ) (Figure. 03).

The ANOVA revealed no significant main effect of GENOTYPE $\left(F_{1,41}=0.88 ; p=0.35\right)$, and GENOTYPE $x$ TIME interaction $\left(F_{4,164}=0.74 ; p=0.56\right)$. The effect of TIME was significant $\left(F_{4,164}=8.14 ; p=0.0005\right)$.

tACS: According to the t-test there was a significant increase in MEP size after tACS in both groups but the excitability enhancement was more pronounced in the Val66Val group (Val66Val group: at 10 and 30 min: 3.4<t>3.86, $p<0.02$; Val66Met group: only at $10 \mathrm{~min} t=3.27, p=0.02$ ) (Figure. 04).

The ANOVA revealed no significant main effect of GENOTYPE $\left(F_{1,11}=0.76 ; p=0.4\right)$, TIME $\left(F_{4,44}=2.13 ; p=0.09\right)$ and GENOTYPE x TIME interaction $\left(F_{4,44}=0.91 ; p=0.46\right)$.

We observed that although we had a higher number of Val66Met subject in the iTBS and tDCS groups than according the sample size calculation should be, the GENOTYPE x TIME interactions remained non-significant. According to the power analysis based on the mean differences of the present data (alpha set at 0.05) a sample size (Val66Met) of 18 a-tDCS, 17 c-tDCS, 12 tACS, 15 iTBS would be necessary.

\section{Comparing the excitability enhancing protocols}

The ANOVA revealed no significant main effect of GENOTYPE $\left(F_{1,103}=0.82 ; p=0.34\right)$, and there were no significant GENOTYPE x STIMULATION $\left(F_{3,103}=1.12 ; p=0.35\right)$ and GENOTYPE x STIMULATION x TIME interactions $\left(F_{12,412}=0.80 ; p=0.65\right)$. There was a significant main effect of STIMULATION $\left(F_{3,103}=5.65 ; p=0.001\right)$ and the STIMULATION x TIME interaction was also significant $\left(F_{12,412}=1.8 ; p=0.05\right)$ (Figure. 05).

Cite as: Antal A, Chaieb L, Moliadze V, et al. BDNF Gene Polymorphisms and Motor Cortical Plasticity in Healthy Humans: When Should We Consider It?. J Neurosci Rehabil. 2014;1 (2):1-13. 


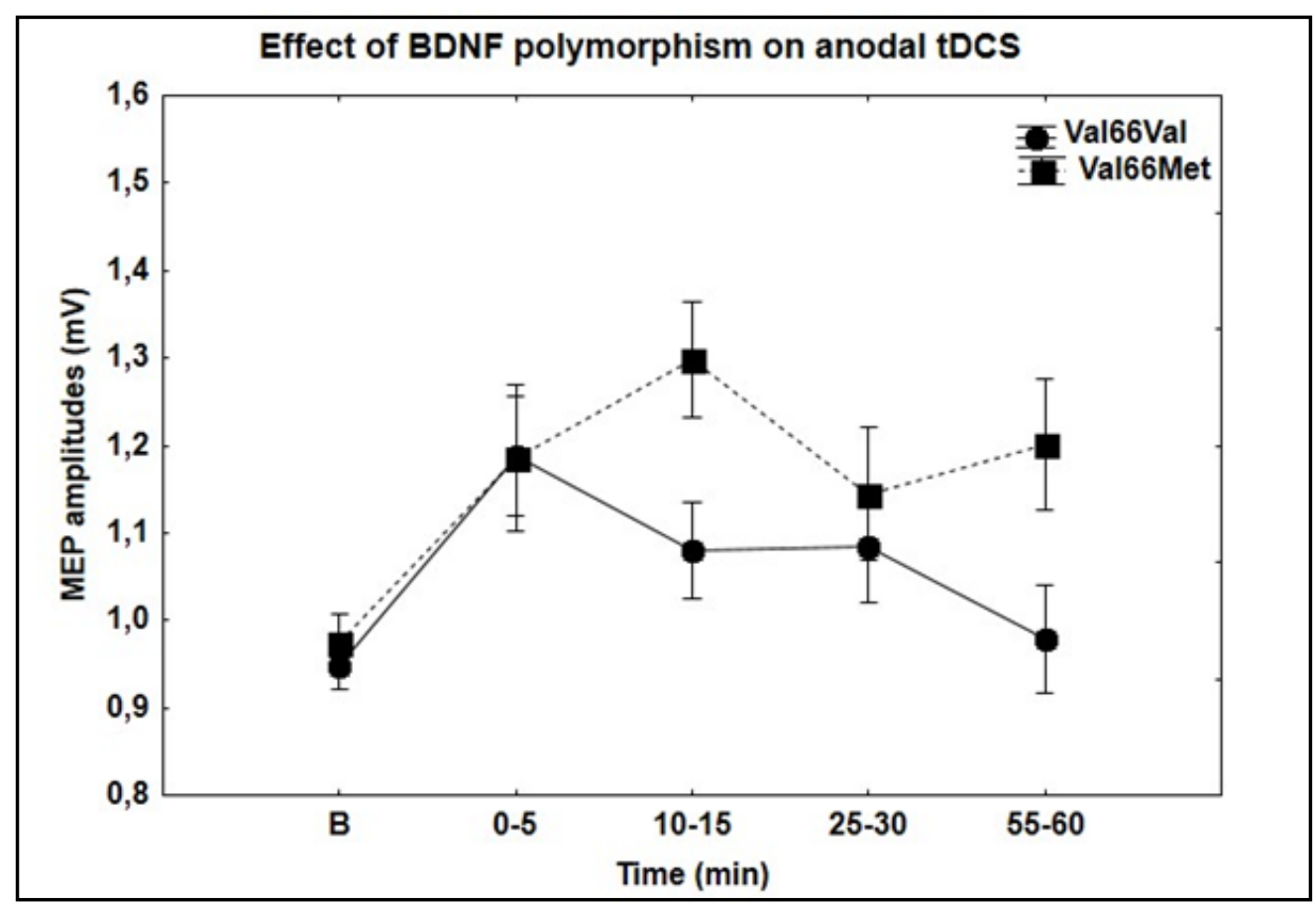

Figure. 02A Effect of BDNF polymorphism on anodal tDCS

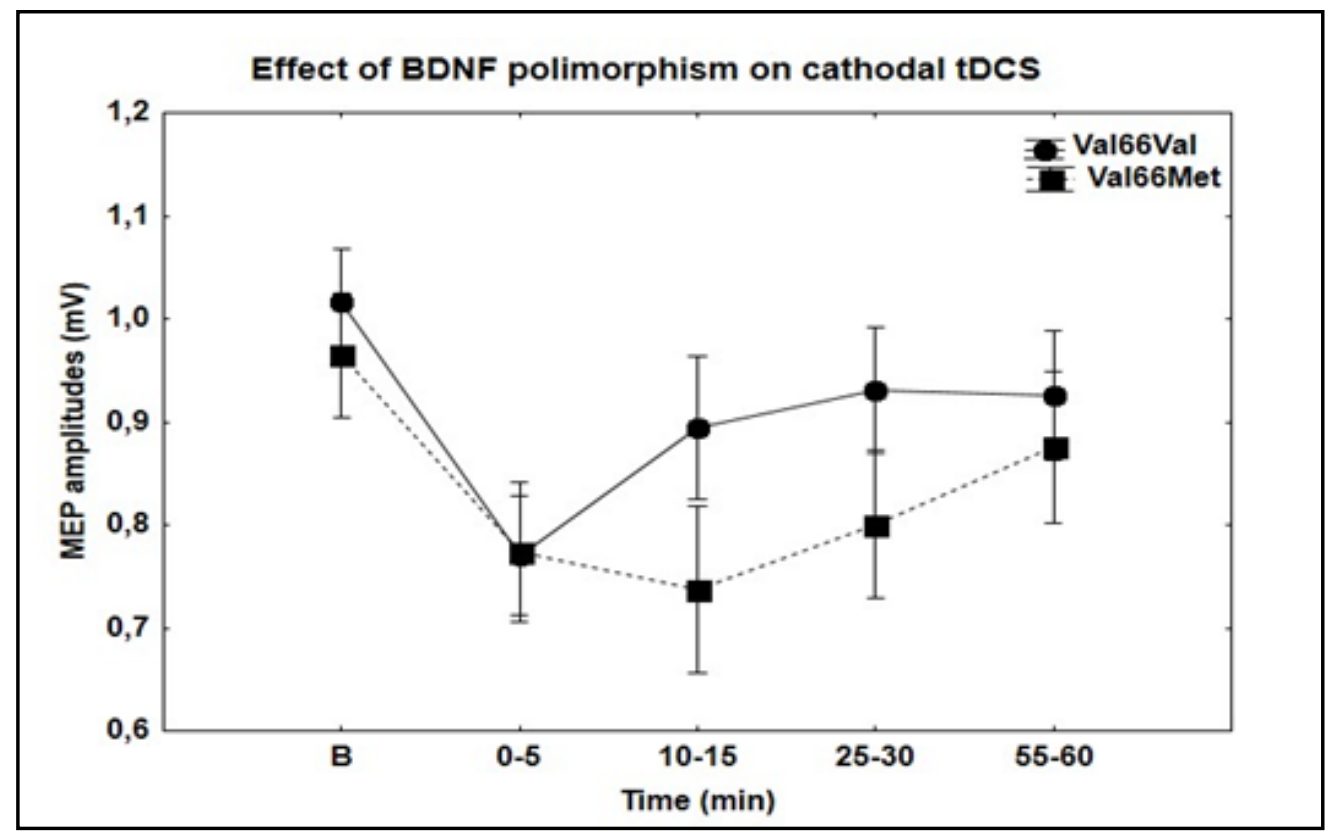

Figure. 02B Effect of BDNF polymorphism on cathodal tDCS

Figure. 02 Effect of BDNF Val66Met polymorphism on cortical excitability in response to cathodal and anodal tDCS. There was an increase in MEP size after anodal tDCS in both groups. With regard to the cathodal effect, there was a significant decrease in MEP amplitudes after cathodal tDCS in both groups. Data are mean (+ S.E.M.) peak-to-peak amplitudes of MEP.

Cite as: Antal A, Chaieb L, Moliadze V, et al. BDNF Gene Polymorphisms and Motor Cortical Plasticity in Healthy Humans: When Should We Consider It?. J Neurosci Rehabil. 2014;1 (2):1-13. 


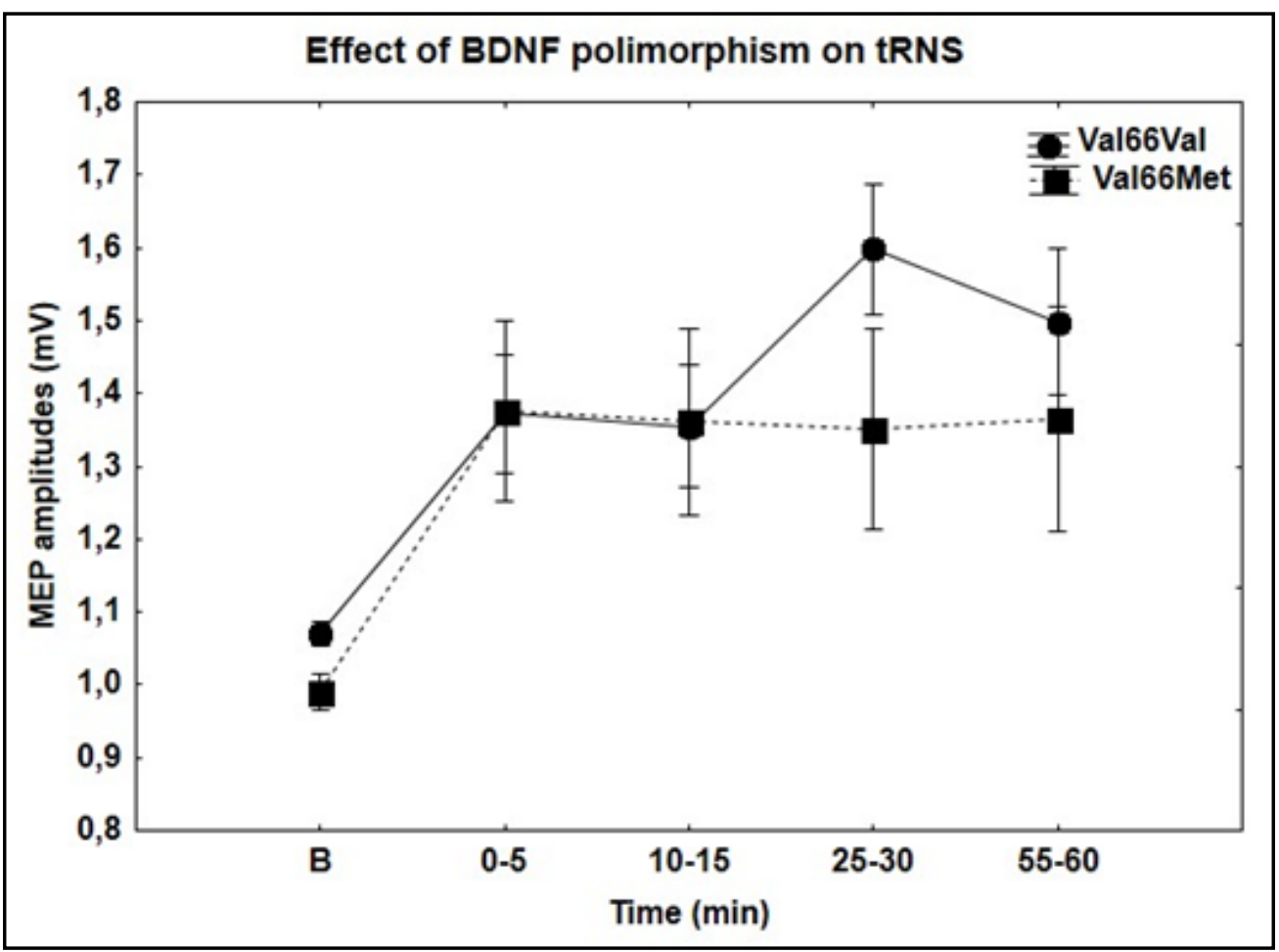

Figure. 03 Effect of BDNF polymorphism on tRNS

Figure. 03 Effect of BDNF Val66Met polymorphism on cortical excitability in response to tRNS. There was a significant increase in MEP amplitude after tRNS in both groups. Data are mean (+ S.E.M.) peak-to-peak amplitudes of MEP.

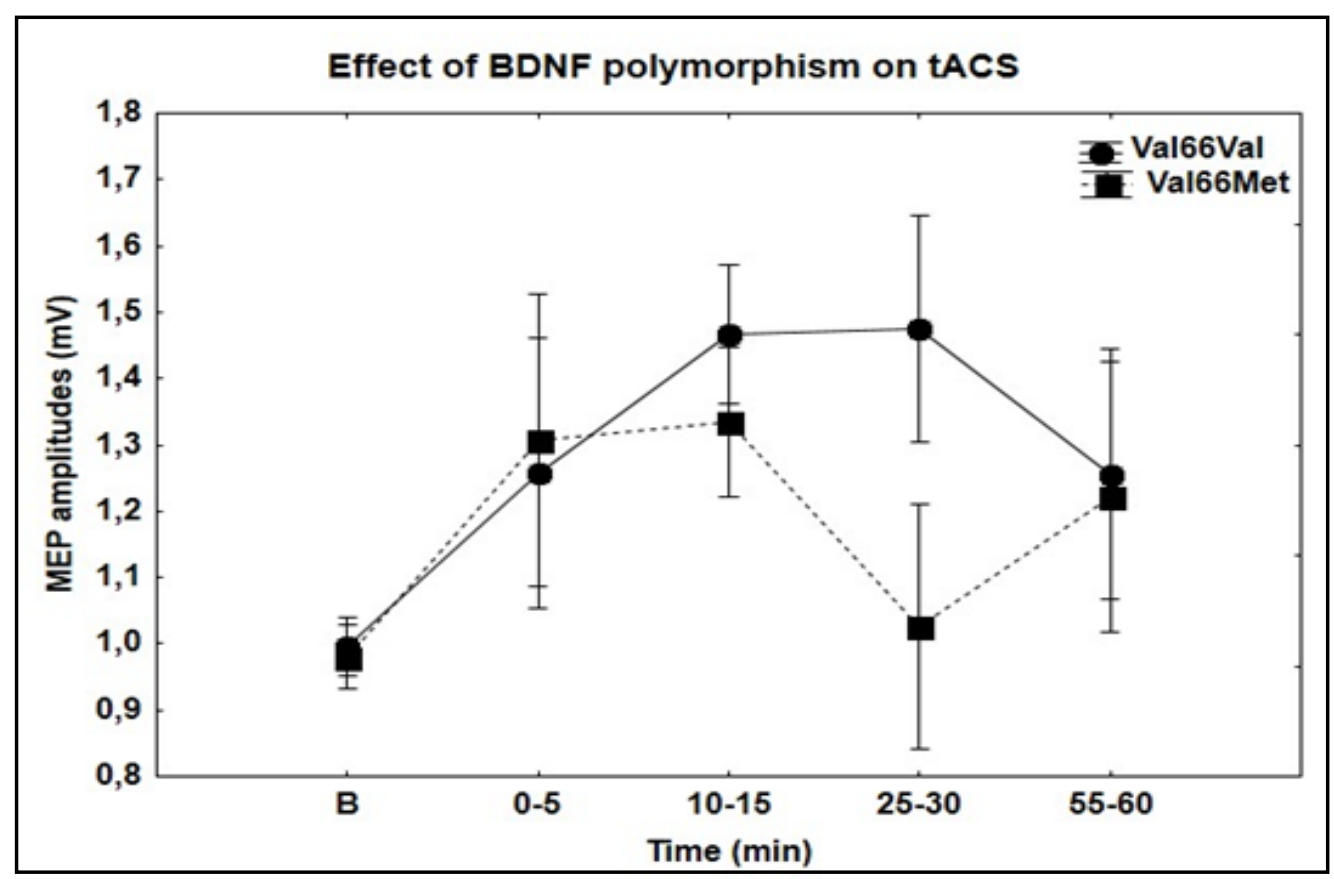

Figure. 04 Effect of BDNF polymorphism on tACS

Cite as: Antal A, Chaieb L, Moliadze V, et al. BDNF Gene Polymorphisms and Motor Cortical Plasticity in Healthy Humans: When Should We Consider It?. J Neurosci Rehabil. 2014;1 (2):1-13. 


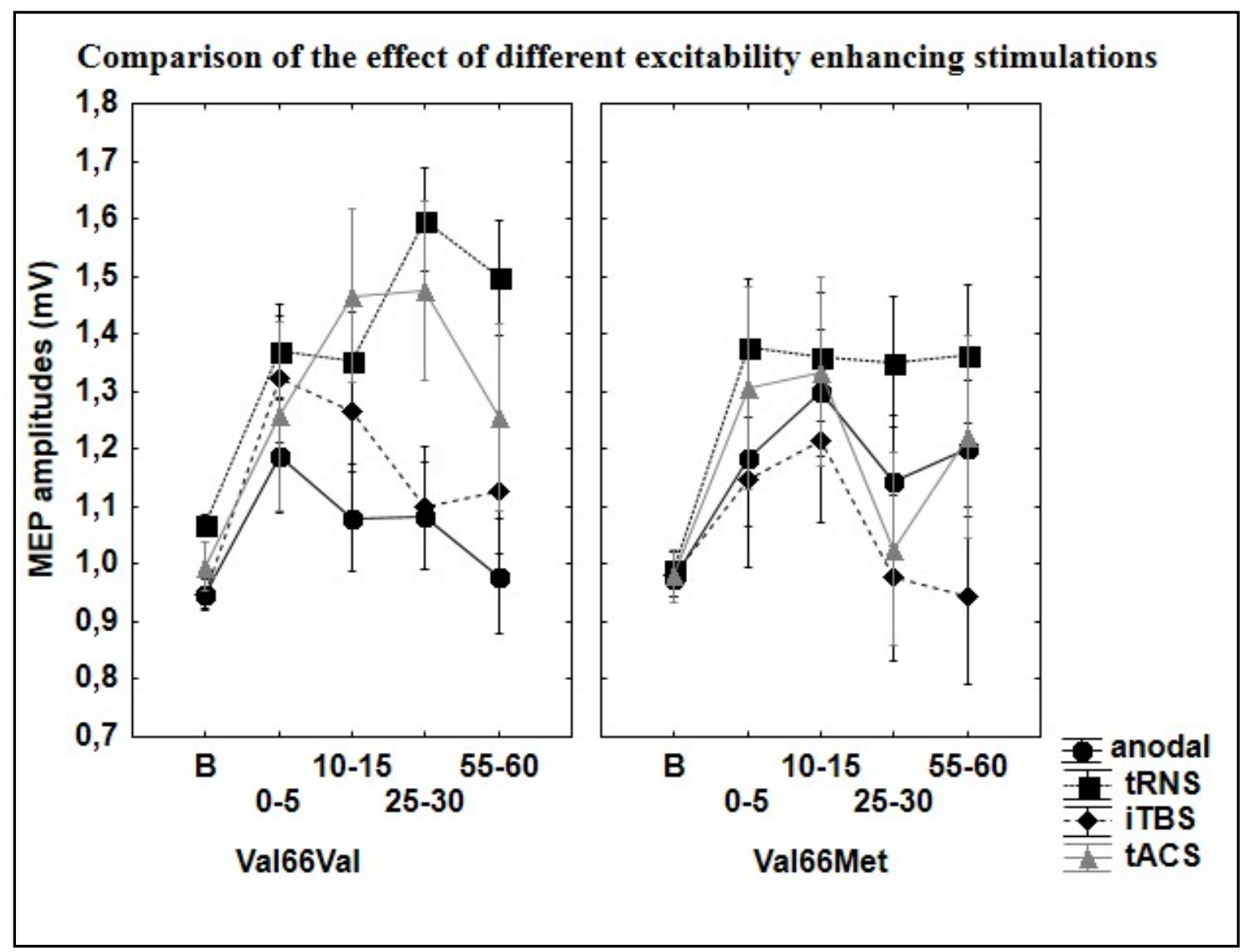

Figure. 05 Comparison of the effect of different excitability enhancing stimulations

Figure. 05 Effect of BDNF Val66Met polymorphism on cortical excitability in response to all of the excitability enhancing conditions. Data are mean (+ S.E.M.) peak-to-peak amplitudes of MEP.

Discussion

In this study we were unable to observe statistically significant effects demonstrating the impact of the BDNF polymorphism on neuroplasticity-inducing methods after comparing a variety of different transcranial stimulation protocols, although some trends were seen. These negative results might be related to the low numbers of subjects in the Val66Met group, although the sample size calculations based on previous results suggested that at least in the iTBS and tDCS groups the number of subjects should be sufficient. According to these data, we have to consider that the association between genotype and the effect of transcranial neuromodulation less important and might be an artifact of small sample sizes. On the whole, the finding of a rather weak BDNF effect is in accordance with previous literature reporting contradictory findings. ${ }^{[10-12,22]}$ Therefore, we suggest that the

Cite as: Antal A, Chaieb L, Moliadze V, et al. BDNF Gene Polymorphisms and Motor Cortical Plasticity in Healthy Humans: When Should We Consider It?. J Neurosci Rehabil. 2014;1 (2):1-13. 
effort given to genotyping subjects for the purpose of examining plasticity-induction in transcranial magnetic or electrical stimulation studies has probably not been rewarded sufficiently by clearer results.

There is no doubt that BDNF plays a very important role in the effects that transcranial stimulation techniques have on the brain. ${ }^{[23-25]}$ Fritsch et al. ${ }^{[23]}$ have measured BDNF levels before and after anodal DCS in rat brain slices and found that combined DCS and low frequency stimulation enhanced BDNF-secretion and tyrosine kinase receptor activation. In another study reduced BDNF expression was found in cathodally stimulated rat brain slices. ${ }^{[2]}$ An interesting question concerns the interaction between BDNF and stimulation duration. It was reported that a longer stimulation duration of $30 \mathrm{~min}$. (such as those used in quadri-pulse stimulation (QPS) paradigms), ${ }^{[12]}$ may be able to overrun BDNF sensitivity, compared to a short TBS protocol lasting between 40 $\mathrm{s}$ and $3 \mathrm{~min}$. This is essentially compatible with a negative finding for a $20 \mathrm{~min}$ tDCS stimulation protocol. ${ }^{[14]}$

tRNS aftereffects appear to be the least affected by BDNF variants (Figure. 03), which is in line with a different physiological mechanism of action. Interestingly, $140 \mathrm{~Hz}$ tACS showed a similar effect to iTBS. We observed that in Val66Val individuals, tACS facilitated MEPs stronger, than in subjects carrying the Met allele, however, this effect was not significant. The working mechanism for this tACS frequency has not yet been ascertained, nevertheless the involvement of GABAA receptors have been implicated. ${ }^{[16]}$ On the cellular level, BDNF secretion is altered by the application of electrical currents, and it has been shown that combined direct current stimulation and low frequency stimulation enhances BDNF-secretion and TrkB activation. ${ }^{[18]}$ While mechanisms of neuroplasticity induction involve the potentiation of NMDA receptors ${ }^{[26]}$ amongst other neurotransmitters, it may be plausible that either transcranial stimulation methods alter BDNF secretion, or that altered levels of BDNF under targetted areas can impact upon the inducible aftereffects of these stimulation methods.

One of the limitations of our study is that it is a retrospective analysis and therefore the number, the age and the male/female distribution of the homo- and heterozygote groups could not exactly be matched. Although our subjects were within the same age-range, the ratio of females/males was slightly different between the two populations. Our Gender $x$ Genotype analysis did not result in significant interaction $(p=0.6)$. The socioeconomic background of the subjects was not explored and the educational level of the subject was close to the same, $90 \%$ of the subjects were university students. Therefore, the current results have to be replicated and validated in a dedicated prospective study. Furthermore, the results described here may not be widely applicable with regard to the stimulation of other areas or using other type of tasks. Previous findings from different groups ${ }^{[27-30]}$ have found bilateral reduction of hippocampal gray matter volumes in Met BDNF-carriers compared with Val66Val subjects, although later investigations have failed to reproduce this finding. ${ }^{[31,32]}$ It has also described that Met carriers have smaller parahippocampal areas and a smaller thalamus compared to Val/ Val subjects. Additional loci of reduced gray matter volumes were found in the frontal areas, including the lateral convexity of the frontal lobes with peak values encompassing the dorsolateral prefrontal cortex bilaterally. ${ }^{[27,28]}$ Accordingly, cognitive studies investigating hippocampus-dependent declarative memory functions (especially concerning paradigms measuring episodic memory) have shown altered memory recall, reporting an association between a decrease in episodic memory function in Met carriers, when compared to Val homozygotes. [27,33-35]

Furthermore, it is also not yet known, whether results derived from studies conducted on healthy populations can be directly translated to patient populations. A study investigated the role of the BDNF polymorphism in response to rTMS in patients affected by mood disorders. ${ }^{[36]}$ rTMS treatment improved depression symptomatology and the response was significantly greater in Val homozygotes than in Met allele carriers. Nevertheless,

Cite as: Antal A, Chaieb L, Moliadze V, et al. BDNF Gene Polymorphisms and Motor Cortical Plasticity in Healthy Humans: When Should We Consider It?. J Neurosci Rehabil. 2014;1 (2):1-13. 
in another study no effect of BDNF polymorphism was observed when depressive patients with anodal tDCS were treated. ${ }^{[37]}$ Yet these results have limited generalizability, because we have no information concerning other diseases.

Recently, the interaction between BDNF Val/Met and COMT Val/Met genotypes on paired associative stimula-

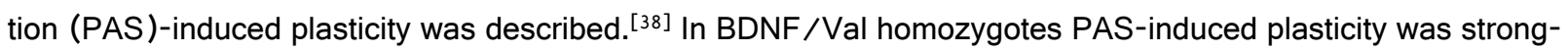
er if participants were also COMT/Met homozygotes, compared with BDNF/Met carriers. Therefore, it is very likely that additional genes and polymorphisms can also influence the effect of transcranial stimulation methods.

\section{ACKNOWLEDGEMENTS}

We would like to thank Drs. K. Monte-Silva, C. Poreisz, N. Thirugnanasambandam, MA. Nitsche, H. Ludwig in participating in the previous version of this work and providing us with electrophysiological data.

\section{AbBreviations}

ADM: Abductor Digiti Minimi

AMT: Active Motor Threshold

BDNF: Brain-Derived Neurotrophic Factor

EMG: Electromyography

FDI: First Dorsal Interosseous

iTBS: Intermittent Theta Burst Stimulation

LTD: Long-Term Depression

LTP: $\quad$ Long-Term Potentiation

M1: Primary Motor Cortex

MEP: Motor-Evoked Potential

PAS: Paired Associative Stimulation

rTMS: Repetitive Transcranial Magnetic Stimulation

tACS: Transcranial Alternating Current Stimulation

TBS: Theta Burst Stimulation

tDCS: Transcranial Direct Current Stimulation

TMS: Transcranial Magnetic Stimulation

tRNS: Transcranial Random Noise Stimulation

\section{REFERENCES}

1. Hanson IM, Seawright A, van Heyningen V. The human BDNF gene maps between FSHB and HVBS1 at the boundary of 11p13-p14. Genomics. 1992;13(4):1331-1333.

2. Lu J, Wu Y, Sousa N, et al. SMAD pathway mediation of BDNF and TGF beta 2 regulation of proliferation and differentiation of hippocampal granule neurons. Development. 2005;132(14):3231-3242.

3. Aiyagari AL, Taylor BR, Aurora $\mathrm{V}$, et al. Hematologic effects of inactivating the Ras processing enzyme Rce1. Blood. 2003;101(6):2250-2252.

4. Lu B. Pro-region of neurotrophins: role in synaptic modulation. Neuron. 2003;39(5):735-738.

5. Lu B. BDNF and activity-dependent synaptic modulation. Learning \& memory. 2003;10(2):86-98.

Cite as: Antal A, Chaieb L, Moliadze V, et al. BDNF Gene Polymorphisms and Motor Cortical Plasticity in Healthy Humans: When Should We Consider It?. J Neurosci Rehabil. 2014;1 (2):1-13. 
6. Jeanneteau F, Chao MV. Promoting neurotrophic effects by GPCR ligands. Novartis Foundation symposium. 2006;276:181-189.

7. Levi-Montalcini R. The nerve growth factor 35 years later. Science. 1987;237(4819):1154-1162.

8. Cheeran $\mathrm{B}$, Talelli $\mathrm{P}$, Mori $\mathrm{F}$, et al. A common polymorphism in the brain-derived neurotrophic factor gene (BDNF) modulates human cortical plasticity and the response to rTMS. J physiology. 2008;586(Pt 23):5717-5725.

9. Antal A, Chaieb L, Moliadze V, et al. Brain-derived neurotrophic factor (BDNF) gene polymorphisms shape cortical plasticity in humans. Brain stimul. 2010;3(4):230-237.

10. Li Voti P, Conte A, Suppa A, et al. Correlation between cortical plasticity, motor learning and BDNF genotype in healthy subjects. Exp Brain Res. 2011;212(1):91-99.

11. Mastroeni C, Bergmann TO, Rizzo V, et al. Brain-derived neurotrophic factor--a major player in stimulation-induced homeostatic metaplasticity of human motor cortex? PloS one. 2013;8(2):e57957.

12. Nakamura K, Enomoto H, Hanajima R, et al. Quadri-pulse stimulation (QPS) induced LTP/LTD was not affected by Val66Met polymorphism in the brain-derived neurotrophic factor (BDNF) gene. Neurosci Lett. 2011;487(3):264-267.

13. Chaieb L, Antal A, Ambrus GG, et al. Brain-derived neurotrophic factor: its impact upon neuroplasticity and neuroplasticity inducing transcranial brain stimulation protocols. Neurogenetics. 2014.

14. Di Lazzaro V, Manganelli F, Dileone M, et al. The effects of prolonged cathodal direct current stimulation on the excitatory and inhibitory circuits of the ipsilateral and contralateral motor cortex. $\mathrm{J}$ neural transm. 2012;119(12):1499-1506.

15. Terney $\mathrm{D}$, Chaieb L, Moliadze $\mathrm{V}$, et al. Increasing human brain excitability by transcranial high-frequency random noise stimulation. J Neurosci. 2008;28(52):14147-1455.

16. Moliadze V, Antal A, Paulus W. Boosting brain excitability by transcranial high frequency stimulation in the ripple range. J physiol. 2010;588(Pt 24):4891-4904.

17. Moliadze V, Atalay D, Antal A, et al. Close to threshold transcranial electrical stimulation preferentially activates inhibitory networks before switching to excitation with higher intensities. Brain stimul. 2012;5(4):505511.

18. Monte-Silva K, Kuo MF, Liebetanz D, et al. Shaping the optimal repetition interval for cathodal transcranial direct current stimulation (tDCS). J neurophysiol. 2010;103(4):1735-1740.

19. Gamboa OL, Antal A, Laczo B, et al. Impact of repetitive theta burst stimulation on motor cortex excitability. Brain stimul. 2011;4(3):145-151.

20. Gamboa OL, Antal A, Moliadze V, et al. Simply longer is not better: reversal of theta burst after-effect with prolonged stimulation. Exp brain res. 2010;204(2):181-187.

21. Antal A, Terney D, Poreisz C, et al. Towards unravelling task-related modulations of neuroplastic changes induced in the human motor cortex. Eur J Neurosci. 2007;26(9):2687-2691.

22. Jayasekeran V, Pendleton N, Holland G, et al. Val66Met in brain-derived neurotrophic factor affects stimulus-induced plasticity in the human pharyngeal motor cortex. Gastroenterology. 2011;141(3):827-36 e1-3.

Cite as: Antal A, Chaieb L, Moliadze V, et al. BDNF Gene Polymorphisms and Motor Cortical Plasticity in Healthy Humans: When Should We Consider It? J J Neurosci Rehabil. 2014;1 (2):1-13. 
23. Fritsch B, Reis J, Martinowich K, et al. Direct current stimulation promotes BDNF-dependent synaptic plasticity: potential implications for motor learning. Neuron. 2010;66(2):198-204.

24. Ranieri F, Podda MV, Riccardi E, et al. Modulation of LTP at rat hippocampal CA3-CA1 synapses by direct current stimulation. J Neurophysiol. 2012;107(7):1868-1880.

25. Lee JY, Kim SH, Ko AR, et al. Therapeutic effects of repetitive transcranial magnetic stimulation in an animal model of Parkinson's disease. Brain res. 2013;1537:290-302.

26. Liebetanz D, Nitsche MA, Tergau F, et al. Pharmacological approach to the mechanisms of transcranial DC-stimulation-induced after-effects of human motor cortex excitability. Brain. 2002;125(Pt 10):22382247.

27. Egan MF, Kojima M, Callicott JH, et al. The BDNF val66met polymorphism affects activity-dependent secretion of BDNF and human memory and hippocampal function. Cell. 2003;112(2):257-269.

28. Pezawas L, Verchinski BA, Mattay VS, et al. The brain-derived neurotrophic factor val66met polymorphism and variation in human cortical morphology. J Neurosci. 2004;24(45):10099-102.

29. Bueller JA, Aftab M, Sen S, et al. BDNF Val66Met allele is associated with reduced hippocampal volume in healthy subjects. Biol Psychiatry. 2006;59(9):812-815.

30. Montag C, Weber B, Fliessbach K, et al. The BDNF Val66Met polymorphism impacts parahippocampal and amygdala volume in healthy humans: incremental support for a genetic risk factor for depression. Psychol med. 2009;39(11):1831-1839.

31. Karnik MS, Wang L, Barch DM, et al. BDNF polymorphism rs6265 and hippocampal structure and memory performance in healthy control subjects. Psychiatry Res. 2010;178(2):425-429.

32. Richter-Schmidinger $\mathrm{T}$, Alexopoulos $\mathrm{P}$, Horn $\mathrm{M}$, et al. Influence of brain-derived neurotrophic-factor and apolipoprotein $\mathrm{E}$ genetic variants on hippocampal volume and memory performance in healthy young adults. J Neural Transm. 2011;118(2):249-257.

33. Hariri AR, Goldberg TE, Mattay VS, et al. Brain-derived neurotrophic factor val66met polymorphism affects human memory-related hippocampal activity and predicts memory performance. J Neurosci. 2003;23(17):6690-6694.

34. Dempster E, Toulopoulou T, McDonald C, et al. Association between BDNF val66 met genotype and episodic memory. Am J Med Genet B Neuropsychiatr Genet. 2005;134B(1):73-75.

35. Kambeitz JP, Bhattacharyya S, Kambeitz-Ilankovic LM, et al. Effect of BDNF val(66)met polymorphism on declarative memory and its neural substrate: a meta-analysis. Neurosci Biobehav Rev. 2012;36(9):21652177.

36. Bocchio-Chiavetto L, Miniussi C, Zanardini R, et al. 5-HTTLPR and BDNF Val66Met polymorphisms and response to rTMS treatment in drug resistant depression. Neurosci lett. 2008;437(2):130-134.

37. Brunoni AR, Kemp AH, Shiozawa P, et al. Impact of 5-HTTLPR and BDNF polymorphisms on response to sertraline versus transcranial direct current stimulation: implications for the serotonergic system. Eur Neuropsychopharmacol 2013;23(11):1530-1540.

38. Witte AV, Kurten J, Jansen S, et al. Interaction of BDNF and COMT polymorphisms on paired-associative stimulation-induced cortical plasticity. J Neurosci. 2012;32(13):4553-4561.

Cite as: Antal A, Chaieb L, Moliadze V, et al. BDNF Gene Polymorphisms and Motor Cortical Plasticity in Healthy Humans: When Should We Consider It?. J Neurosci Rehabil. 2014;1 (2):1-13. 